\title{
Serum HBsAg and HBeAg levels are associated with liver pathological stages in the immune clearance phase of hepatitis B virus chronic infection
}

\author{
DA-WU ZENG ${ }^{*}$, YU-RUI LIU*, JING DONG, YUE-YONG ZHU, \\ YOU-BING LI, JING CHEN, QI ZHENG and JIA-JI JIANG \\ Liver Center, First Affiliated Hospital, Fujian Medical University, Fuzhou, Fujian 350005, P.R. China
}

Received November 13, 2013; Accepted May 28, 2014

DOI: $10.3892 / \mathrm{mmr} .2015 .3207$

\begin{abstract}
The association between hepatitis B surface antigen (HBsAg) and hepatitis B 'e' antigen (HBeAg) levels and liver inflammation and fibrosis in patients with chronic hepatitis $\mathrm{B}(\mathrm{CHB})$ in the immune clearance (IC) remains elusive. The aim of the present study was to investigate whether HBsAg and $\mathrm{HBeAg}$ levels were associated with liver inflammation and fibrosis in $\mathrm{CHB}$ patients during the IC phase. Kendall's rank correlation analysis and receiver operating characteristic curves were used to determine the correlation between $\mathrm{HBsAg}$, $\mathrm{HBeAg}$ and liver pathological stages. Multivariate analysis by forward logistic regression was used to analyze significant predictors of cirrhosis. A liver pathology-predicting model (IC model), which used routinely assessed markers in combination with $\mathrm{HBsAg}$ and HBeAg levels, was constructed. There were significantly positive correlations between the HBsAg and HBeAg levels $(\gamma=0.317, \mathrm{P}<0.001)$, and between the HBsAg and HBV-DNA levels $(\gamma=0.489, \mathrm{P}<0.001)$. However, there was no correlation between the HBsAg and alanine aminotransferase levels. HBsAg and HBeAg levels differed significantly at various liver pathological stages and declined progressively in advanced liver pathological stages. Multivariate logistic regression analysis showed that age, $\mathrm{HBs} \mathrm{Ag}$ and $\mathrm{HBeAg}$ levels as well as the international normalized ratio (INR) were independent predictors of liver fibrosis during the IC phase. The IC model had a specificity and sensitivity of 88.64 and $78.24 \%$, respectively, a positive predictive value of $48.15 \%$ and negative predictive value of $96.79 \%$. In conclusion, $\mathrm{HBsAg}$ and $\mathrm{HBeAg}$ levels were negatively and indirectly correlated
\end{abstract}

Correspondence to: Dr Jing Dong, Liver Center, First Affiliated Hospital, Fujian Medical University, 20 Chazhong Road, Taijiang District, Fuzhou, Fujian 350005, P.R. China

E-mail: drdongj@yeah.net

*Contributed equally

Key words: biopsy, chronic hepatitis B, fibrosis, HBsAg, HBeAg, immune clearance with liver inflammation and fibrosis in CHB patients in the IC phase. The IC model reliably predicted the probability of liver cirrhosis.

\section{Introduction}

Chronic hepatitis B virus (CHB) represents a serious global health concern. The World Health Organization (WHO) estimates that there are 400 million chronic HBV carriers worldwide, and the weighted prevalence of hepatitis B surface antigen (HBsAg) for the Chinese population aged 1-59 years was shown to be $7.18 \%$ (1). Patients with $\mathrm{CHB}$ have a higher risk of developing liver cirrhosis, hepatic decompensation and hepatocellular carcinoma, and $15-20 \%$ of patients develop liver cirrhosis within five years (2).

The natural course of CHB includes: i) An immunotolerance (IT) phase characterized by the presence of hepatitis $B$ 'e' antigen (HBeAg), active HBV replication and normal levels of alanine aminotransferase (ALT), ii) an immunoclearance (IC) phase characterized by fluctuating or high serum HBV DNA and ALT levels, liver inflammation and HBeAg seroconversion, iii) a low replicative (LR) phase in which patients have undetectable levels of HBV DNA, are HBeAg-negative, anti-HBeAg positive and show minimal fibrosis and iv) $\mathrm{HBeAg}$ negative hepatitis (ENH) (3).

Overt HBV infection is characterized by the presence of HBsAg, which is secreted by infected hepatocytes $(4,5)$. HBsAg levels have been suggested to i) reflect the presence of covalently closed circular DNA (cccDNA), ii) predict HBsAg seroclearance and iii) predict the therapeutic response during antiviral therapy (6,7). Although HBsAg levels have traditionally been estimated using enhanced chemiluminescence (8), there has been a recent focus on developing reproducible, automated, low-cost assay systems in order to use it as a biomarker (9).

$\mathrm{HBeAg}$ is an important early serum marker of $\mathrm{HBV}$ infection and correlates with high infectivity (10). Perinatal transmission of $\mathrm{HBV}$ is strongly associated with $\mathrm{HBeAg}$ positivity in the mother (11) and HBeAg seroconversion is considered as a marker for a sustained therapeutic response (12). Quantitative assays for $\mathrm{HBeAg}$ are not routinely available, and HBeAg levels are expressed as serial dilutions 
of a reference sample from the Paul Ehrlich Institute (Langen, Germany) (13). It has been suggested that the evaluation of the HBeAg sample to cut-off ratio (S/co ratio) could be useful for HBV diagnosis and treatment $(12,14)$.

Assessment of fibrosis by liver biopsy remains the most reliable parameter in determining appropriate treatment options (15). Given the invasiveness of the liver biopsy procedure, it has become important to develop novel, non-invasive strategies to diagnose fibrosis. Median HBsAg levels have been shown to fluctuate through the different stages of $\mathrm{HBV}$ infection $(7,9,16)$ and serum HBsAg levels were shown to correlate with HBV DNA levels (17). However, there is limited information describing the association between $\mathrm{HBs} A g$ and liver pathology in patients with CHB who are in the IC phase (18). There is also limited information on the association of $\mathrm{HBeAg}$ with the grade of inflammation and fibrosis in $\mathrm{CHB}$ patients (19). Treatment-naïve HBeAg-positive CHB patients were recently shown to have a strong positive correlation between HBsAg and HBV DNA levels and a negative correlation between HBsAg levels and severity of fibrosis (20). The major goals of this study were to understand the association between $\mathrm{HBsAg}, \mathrm{HBeAg}$ and fibrosis and to evaluate $\mathrm{HBsAg}$ and $\mathrm{HBeAg}$ as non-invasive biomarkers of liver inflammation and fibrosis in CHB patients in the IC phase of the disease.

\section{Patients and methods}

Patients. This study received approval from the Institutional Review Board of Fujian Medical University, Fuzhou, China. Patients' informed consent was obtained prior to the study. This retrospective cross-sectional study included a total of 237 patients diagnosed with CHB and consecutively admitted to the First Affiliated Hospital of Fujian Medical University (Fujian, China) between March 2009 and May 2012. Only patients in the immune clearance phase were included in this study. The IC phase was defined as the presence of HBsAg for at least six months and serum HBV DNA $\geq 2,000 \mathrm{IU} / \mathrm{ml}$ $\left(\sim 10^{4}\right.$ copies $\left./ \mathrm{ml}\right)$ in HBeAg-positive patients. ALT levels at least twice the upper limit of normal (ULN) were used as one of the criteria to define the IC phase, based on previous reports $(6,7,20)$ and based on the APASL guide $(21)$, which defines minimally elevated ALT as a value between ULN and 2X ULN. Exclusion criteria were i) presence of other types of viral hepatitis, ii) hepatocellular carcinoma, iii) alcoholic liver disease, iv) decompensated cirrhosis, v) autoimmune hepatitis, vi) concurrent infection with human immunodeficiency virus (HIV), vii) hereditary liver diseases, viii) drug-induced liver injury and ix) serum creatinine levels 1.5 -fold the ULN. None of the patients received antiviral therapy prior to the liver biopsy. Biochemical and virological data were obtained from patient serum samples collected within the previous seven days.

Liver histology and quantification of fibrosis. Liver tissue was obtained by ultrasonographic-guided, percutaneous liver biopsy. Specimens of 15-20 mm liver tissues were fixed, paraffin-embedded and stained with hematoxylin-eosin-safran and Masson's trichrome. A minimum of six portal tracts was required for diagnosis. The liver biopsies were evaluated with or without knowing the patient history. Histological staging was performed in order to classify the degree of fibrosis according to the Chinese Guidelines of the Programme of Prevention and Cure for Viral Hepatitis (20). All biopsy samples were classified as inflammation levels G $0-4$. This classification system for fibrosis stages (FS) was similar to the Scheuer system (22) and METAVIR system (23), with F0 (no fibrosis), F1 (mild fibrosis without septa), F2 (moderate fibrosis with few septa), F3 (severe fibrosis with numerous septa without cirrhosis) and F4 (cirrhosis) (23). However, in the present study, fibrosis stages were simplified as F1 (F0-F1), F2, F3 and F4.

Serum parameters. Serum HBsAg was quantified using the chemiluminescent ARCHITECT platform (Abbott Laboratories, Chicago, IL, USA), according to the manufacturer's instructions. The ARCHITECT quantitative HBsAg assay is a chemiluminescent microparticle assay, internally calibrated using the WHO standard for HBsAg. Samples were diluted $1: 100$ in horse serum and if $>250 \mathrm{IU} / \mathrm{ml}$, samples were retested at a dilution of 1:500. Serum $\mathrm{HBeAg}$ levels were measured using the AxSYM microparticle enzyme immunoassay (Abbott Laboratories) according to the manufacturer's instructions. The AxSYM assay measures the ratio of the sample (S) to the cut-off $(\mathrm{Co})(\mathrm{S} / \mathrm{Co}$ ratio) and an $\mathrm{S} / \mathrm{Co}$ ratio $\geq 1.0$ is defined as $\mathrm{HBeAg}$-positive. Serum HBV DNA levels were measured by fluorescence quantitative polymerase chain reaction with a detection range of 500-1.0x $10^{9} \mathrm{IU} / \mathrm{ml}$ (HBV PCR Detection kit; PG Company, Shenzhen, China), according to the manufacturer's instructions. Serum biochemical parameters, including total bilirubin, ALT, aspartate aminotransferase (AST), gamma glutamyl transpeptidase (GGT), albumin, globulin, total cholesterol, $\alpha$-fetoprotein, prothrombin time (PT), international normalized ratio (INR), white blood corpuscle count (WBC) and platelet count (PLT) were determined within 1 week prior to taking the liver biopsy.

Model development. Numerous biochemical markers, including albumin, cholinesterase, cholesterol, platelet count, AST, ALT, bilirubin, globin, GGT, total bile acid, prothrombin time and INR were evaluated in addition to HBV DNA, HBsAg, and HBeAg levels, for their ability to determine cirrhosis in CHB patients. These parameters were measured using an Olympus AU2700 Biochemistry Analyzer (Olympus Corporation, Tokyo, Japan). A comparative analysis of patients with or without cirrhosis was then performed in order to select the most appropriate markers to develop a liver pathology-predicting model (IC model). The predictive value of the IC model was compared with other existing models, including the fibrosis index (FI), AST to platelet ratio index (APRI), FIB-4, age-AST and age-platelet (AP) index (24-29) using receiver operating characteristic (ROC) curves.

Statistical analysis. The correlations between clinical parameters and liver pathological stages were determined by using Kendall's rank correlation coefficient, with the exception of correlation between gender and liver pathological stages. Spearman's correlation was used to test the correlation between gender and liver pathological stages. Data are presented as medians (IQR), unless indicated. All continuous variables were analyzed after logarithmic transformation for normality 
Table I. Characteristics of 237 patients in the immune clearance phase of persistent hepatitis B viral-infection and the correlation between clinical parameters and liver pathological stages.

\begin{tabular}{|c|c|c|c|c|c|}
\hline \multirow[b]{2}{*}{ Variables } & \multirow[b]{2}{*}{ Median (IQR) } & \multicolumn{2}{|c|}{ G stage } & \multicolumn{2}{|c|}{ F stage } \\
\hline & & $\begin{array}{l}\text { Correlation } \\
\text { coefficient }\end{array}$ & P-value & $\begin{array}{l}\text { Correlation } \\
\text { coefficient }\end{array}$ & P-value \\
\hline Age (years) ${ }^{\mathrm{a}}$ & $32(24-38)$ & 0.236 & $<0.001^{\mathrm{c}}$ & 0.217 & $<0.001^{\mathrm{c}}$ \\
\hline Gender (male/female) ${ }^{\mathrm{b}}$ & $186 / 51$ & 0.027 & 0.674 & 0.025 & 0.706 \\
\hline Bilirubin $(\mu \mathrm{mol} / \mathrm{l})^{\mathrm{a}}$ & $17(12.7-23.2)$ & 0.145 & $0.004^{\mathrm{c}}$ & 0.137 & $0.005^{\mathrm{c}}$ \\
\hline $\operatorname{Albumin}(\mathrm{g} / \mathrm{l})^{\mathrm{a}}$ & $39.7(36.7-43.2)$ & -0.321 & $<0.001^{\mathrm{c}}$ & -0.268 & $<0.001^{\mathrm{c}}$ \\
\hline Globin $(\mathrm{g} / \mathrm{l})^{\mathrm{a}}$ & $30.5(27.8-33.5)$ & 0.118 & $0.019^{c}$ & 0.128 & $0.008^{\mathrm{c}}$ \\
\hline $\operatorname{ALT}(\mathrm{IU} / 1)^{\mathrm{a}}$ & $226(124-428)$ & 0.108 & $0.031^{\mathrm{c}}$ & 0.022 & 0.646 \\
\hline $\operatorname{AST}(\mathrm{IU} / 1)^{\mathrm{a}}$ & $121(73-228)$ & 0.176 & $<0.001^{\mathrm{c}}$ & 0.094 & 0.054 \\
\hline GGT (IU/l) $)^{\mathrm{a}}$ & $68(43-120)$ & 0.258 & $<0.001^{\mathrm{c}}$ & 0.164 & $0.001^{\mathrm{c}}$ \\
\hline Cholinesterase (IU/l) $)^{\mathrm{a}}$ & $6671(5432-8033)$ & -0.294 & $<0.001^{\mathrm{c}}$ & -0.283 & $<0.001^{\mathrm{c}}$ \\
\hline Total bile acid $(\mathrm{mmol} / \mathrm{l})^{\mathrm{a}}$ & $15.9(8.6-32.8)$ & 0.167 & $0.001^{\mathrm{c}}$ & 0.166 & $0.001^{\mathrm{c}}$ \\
\hline Total cholesterol $(\mathrm{mmol} / \mathrm{l})^{\mathrm{a}}$ & $4.25(3.70-4.98)$ & -0.136 & $0.008^{c}$ & -0.120 & $0.015^{\mathrm{c}}$ \\
\hline Prothrombin time $(\mathrm{s})^{\mathrm{a}}$ & $13.4(12.9-14.3)$ & 0.252 & $<0.001^{\mathrm{c}}$ & 0.271 & $<0.001^{\mathrm{c}}$ \\
\hline $\mathrm{INR}^{\mathrm{a}}$ & $1.02(0.97-1.10)$ & 0.308 & $<0.001^{\mathrm{c}}$ & 0.283 & $<0.001^{\mathrm{c}}$ \\
\hline White cell count $\left(10^{9} / 1\right)^{\mathrm{a}}$ & $5.6(4.6-6.6)$ & -0.128 & $0.011^{\mathrm{c}}$ & -0.053 & 0.277 \\
\hline Platelet count $\left(10^{11} / 1\right)^{\mathrm{a}}$ & $1.91(1.58-2.22)$ & -0.248 & $<0.001^{\mathrm{c}}$ & -0.277 & $<0.001^{\mathrm{c}}$ \\
\hline HBV DNA $(\log \mathrm{IU} / \mathrm{ml})^{\mathrm{a}}$ & $6.81(5.86-7.45)$ & -0.132 & $0.008^{c}$ & -0.130 & $0.008^{\mathrm{c}}$ \\
\hline HBsAg $(\log \mathrm{IU} / \mathrm{ml})^{\mathrm{a}}$ & $3.94(3.45-4.41)$ & -0.244 & $<0.001^{\mathrm{c}}$ & -0.298 & $<0.001^{\mathrm{c}}$ \\
\hline $\mathrm{HBeAg}(\log \mathrm{S} / \mathrm{Co})^{\mathrm{a}}$ & $2.63(2.07-3.01)$ & -0.304 & $<0.001^{\mathrm{c}}$ & -0.371 & $<0.001^{\mathrm{c}}$ \\
\hline
\end{tabular}

P-values are from ${ }^{a}$ Kendall's rank correlation coefficient; ${ }^{b}$ Spearman's correlation coefficient. ${ }^{\text {P }}$-values indicate statistical significance between the clinical parameters and the liver pathological stages. G stage, liver inflammation stage; F stage, liver fibrosis stage; IQR, interquartile range; ALT, alanine aminotransferase; AST, aspartate aminotransferase; GGT, gamma glutamyl transpeptidase; INR, international normalized ratio; HBV, hepatitis B virus.

of distribution. Univariate logistic regression analysis was performed to analyze the odds ratio (OR) of significant factors associated with patients with cirrhosis. Variables with a $\mathrm{P}<0.05$ in the univariate analysis were selected and evaluated by multivariate logistic regression models with a conditional forward selection method. Furthermore, an ROC curve was employed to obtain the area under the curve (AUC), sensitivity, specificity, positive predictive value (PPV) and negative predictive value (NPV) of HBsAg and HBeAg levels, in order to distinguish between the different stages of inflammation and fibrosis. ROC curves were constructed for the IC model, APRI, age-AST, FIB4, FI and the AP index to predict cirrhosis. The AUC was statistically construed as the probability of assessing $\mathrm{HBsAg}$ and $\mathrm{HBeAg}$ as non-invasive biomarkers of liver inflammation and fibrosis in $\mathrm{CHB}$ patients who were in the IC stage of the disease. All statistical assessments were two-sided and evaluated at the 0.05 level of significance. Statistical analyses were performed using SPSS 15.0 statistics software (SPSS, Inc., Chicago, IL, USA). Multiple ROC curves were analyzed to predict cirrhosis with six non-invasive models (IC-model, FI, APRI, FIB-4, age-AST model and AP index). For pairwise comparison of the ROC curves, MedCalc for Windows, version 9.38 (MedCalc Software, Mariakerke, Belgium), was used.

\section{Results}

The clinical characteristics of CHB patients in the IC phase and the correlation between the clinical characteristics and liver pathological stages are summarized in Table I. Two hundred and thirty seven patients admitted to the First Affiliated Hospital of Fujian Medical University consecutively, and diagnosed with CHB from March 2009 to May 2012, were included in this cross-sectional study. Of the 237 patients, $186(78.5 \%)$ were male and the median age of all patients was 32 years (range, 11-61 years). 54 (22.8\%), 78 (32.9\%), 61 (25.7\%) and $44(18.6 \%)$ had F1, F2, F3 or F4 fibrosis, respectively. The levels of albumin, cholinesterase, cholesterol, platelet count, HBV DNA, HBsAg and HBeAg were negatively correlated with liver pathological stages (both the inflammation and fibrosis stages). In contrast, age, bilirubin, globin, GGT, total bile acid, prothrombin time and international normalized ratio (INR) were positively correlated with liver pathological stages (Table I). There were significant positive correlations between $\mathrm{HBs}$ Ag and HBeAg levels $(\gamma=0.317, P<0.001)$, and between HBsAg and HBV-DNA levels $(\gamma=0.489, \mathrm{P}<0.001)$. However, no correlation between HBsAg and ALT levels was identified (data not shown). 
Table II. HBsAg and HBeAg levels distinguish different stages of inflammation and fibrosis as measured by AUC values .

\begin{tabular}{lccccc}
\hline & AUC $(95 \% \mathrm{CI})$ & Youden index & Cut-off point & Sensitivity (\%) & Specificity (\%) \\
\hline $\mathrm{HBsAg}$ & & & & & \\
$\mathrm{G} \geq 4$ & $0.73(0.66,0.81)$ & 0.460 & 3.76 & 68.53 & 77.50 \\
$\mathrm{~F} \geq 3$ & $0.72(0.65,0.78)$ & 0.344 & 4.20 & 51.52 & 82.86 \\
$\mathrm{~F} \geq 4$ & $0.74(0.67,0.81)$ & 0.449 & 3.75 & 69.95 & 75.00 \\
$\mathrm{HBeAg}$ & & & & & 85.00 \\
$\mathrm{G} \geq 4$ & $0.77(0.70,0.84)$ & 0.520 & 2.43 & 67.00 & 68.57 \\
$\mathrm{~F} \geq 3$ & $0.77(0.71,0.83)$ & 0.451 & 2.47 & 76.52 & 84.09 \\
$\mathrm{~F} \geq 4$ & $0.78(0.72,0.85)$ & 0.530 & 2.42 & 68.91 & \\
\hline
\end{tabular}

AUC, area under the curve; CI, confidence interval; G, liver inflammation stages; F3, severe fibrosis with numerous septa without cirrhosis; F4, cirrhosis; HBsAg, hepatitis B surface antigen; HBeAg, hepatitis B 'e' antigen.

A $\mathrm{G} \geqq 4$

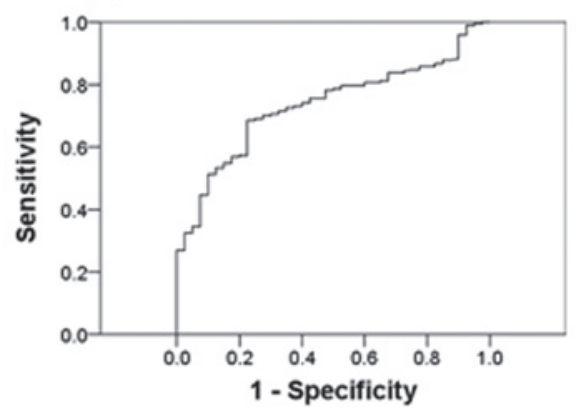

C $\mathrm{F} \geqq 4$

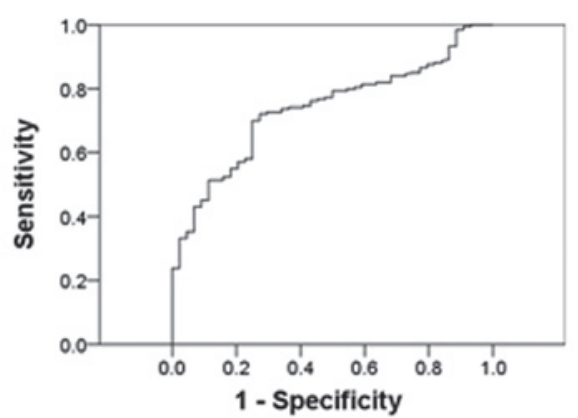

B $\mathrm{F} \geqq 3$

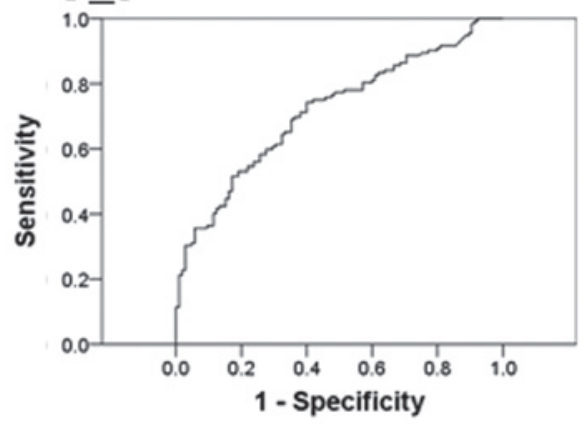

D

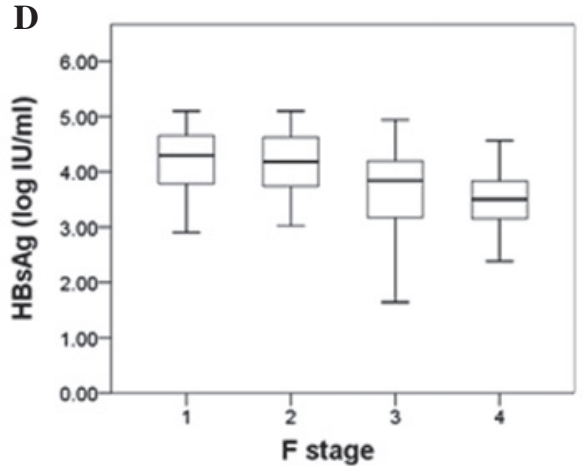

Figure 1. (A-C) Receiver operating characteristic curves of performance of HBsAg levels in distinguishing the different stages of inflammation and fibrosis. (D) Median HBsAg levels $(\log 10 \mathrm{IU} / \mathrm{ml})$ at liver inflammation stages F1 to F4 (1D). G, liver inflammation stage; F, liver firbosis stage; HBsAg, hepatitis B surface antigen; HBeAg, hepatitis B 'e' antigen.

Table II shows the performance of HBsAg and HBeAg levels in distinguishing the different stages of inflammation and fibrosis using AUC values. ROC curve analysis revealed that the AUC was $0.73(\mathrm{G} \geq 4), 0.72(\mathrm{~F} \geq 3)$, and $0.74(\mathrm{~F} \geq 4)$ and the cut-off point of HBsAg $(\log \mathrm{IU} / \mathrm{ml})$ was $3.76,4.20$ and 3.75, respectively (Fig. 1A-C). The median HBsAg levels $(\log 10 \mathrm{IU} / \mathrm{ml})$ at liver fibrosis stages F1 to F4 were 4.29, 4.18, 3.84 and 3.50, respectively (Fig. 1D). Similarly, ROC curve analysis revealed that the area under the curve was $0.77(\mathrm{G} \geq 4), 0.77(\mathrm{~F} \geq 3)$ and $0.78(\mathrm{~F} \geq 4)$, respectively (Fig. 2A-C), and the median HBeAg levels ( $\log 10$ S/Co) were 2.99, 2.91, 2.33 and 2.03, respectively (Fig. 2D).

Table III presents the univariate and multivariate analyses of determinants predicting cirrhosis. The univariate logistic regression model indicated the following significant factors: Age, albumin, globin, GGT, total bile acid, cholesterol, prothrombin time, platelet count, INR, HBsAg, HBeAg, and HBV DNA $(\mathrm{P}<0.05)$. Variables with a $\mathrm{P}<0.05$ in the univariate analysis were selected and evaluated by multivariate logistic regression models using a conditional forward selection method. In addition, prothrombin time was excluded from the final multivariate logistic regression model because of the high correlation between prothrombin and INR. Multivariate logistic regression indicated that age (OR: 1.071, $\mathrm{P}=0.001)$, INR (OR: 10958, $\mathrm{P}<0.001)$, HBsAg (OR: 0.536, $\mathrm{P}=0.009)$ and $\mathrm{HBeAg}(\mathrm{OR}: 0.532, \mathrm{P}=0.013)$ were significantly associated with cirrhosis. Based on these data, 
Table III. Logistic regression analysis to determine factors significantly associated with cirrhosis in patients with chronic hepatitis B virus infection.

\section{Univariate}

Variables

Log Age (years)

Log Bilirubin $(\mu \mathrm{mol} / \mathrm{l})$

Log Albumin (g/l)

Log Globin (g/l)

Log ALT (IU/1)

Log AST (IU/1)

Log GGT (IU/1)

Log Cholinesterase (IU/l)

Log Total bile acid ( $\mathrm{mmol} / \mathrm{l})$

Log Cholesterol (mmol/l)

Prothrombin time (s)

Log White cell count $\left(10^{9} / 1\right)$

Log Platelet count $\left(10^{11} / 1\right)$

Log INR

Log HBsAg (IU/ml)

LogHBeAg (S/Co)

Log HBV DNA (IU/ml)

\begin{tabular}{cccr}
\hline OR $(95 \%$ CI $)$ & P-value & OR $(95 \%$ CI $)$ & P-value \\
\cline { 3 - 4 } $1.077(1.040,1.115)$ & $<0.001^{\mathrm{a}}$ & $1.071(1.027,1.116)$ & $0.001^{\mathrm{a}}$ \\
$1.603(0.985,2.611)$ & 0.058 & & \\
$0.002(0.001,0.046)$ & $<0.001^{\mathrm{a}}$ & & \\
$26.32(2.43,285.17)$ & $0.007^{\mathrm{a}}$ & & \\
$0.977(0.642,1.486)$ & 0.914 & & \\
$1.230(0.814,1.857)$ & 0.326 & & \\
$1.698(1.108,2.600)$ & $0.015^{\mathrm{a}}$ & & \\
$0.427(0.179,1.104)$ & 0.054 & & $<0.001^{\mathrm{a}}$ \\
$1.486(1.081,2.043)$ & $0.015^{\mathrm{a}}$ & & $0.009^{\mathrm{a}}$ \\
$0.116(0.022,0.606)$ & $0.011^{\mathrm{a}}$ & & $0.013^{\mathrm{a}}$ \\
$2.169(1.573,2.990)$ & $<0.01^{\mathrm{a}}$ & & \\
$0.366(0.022,6.168)$ & 0.485 & & \\
$0.033(0.003,0.337)$ & $0.004^{\mathrm{a}}$ & & \\
$5581(159,196075)$ & $<0.001^{\mathrm{a}}$ & $10958(184,652792)$ & \\
$0.463(0.318,0.675)$ & $<0.001^{\mathrm{a}}$ & $0.536(0.323,0.877)$ & \\
$0.381(0.254,0.572)$ & $<0.001^{\mathrm{a}}$ & $0.532(0.323,0.877)$ & \\
$0.773(0.604,0.988)$ & $0.040^{\mathrm{a}}$ & & \\
\hline
\end{tabular}

Univariate logistic regression analysis was performed to analyze the odds ratio of significant factors associated with patients with cirrhosis. Variables having a ${ }^{\mathrm{P}}<0.05$ in the univariate analysis were selected and evaluated by multivariate logistic regression models, with the conditional forward selection method. ALT, alanine aminotransferase; AST, aspartate aminotransferase; GGT, gamma glutamyl transpeptidase; INR, international normalized ratio; OR, odds ratio; HBV, hepatitis B virus; HBsAg, hepatitis B surface antigen; HBeAg, hepatitis B 'e' antigen.

A

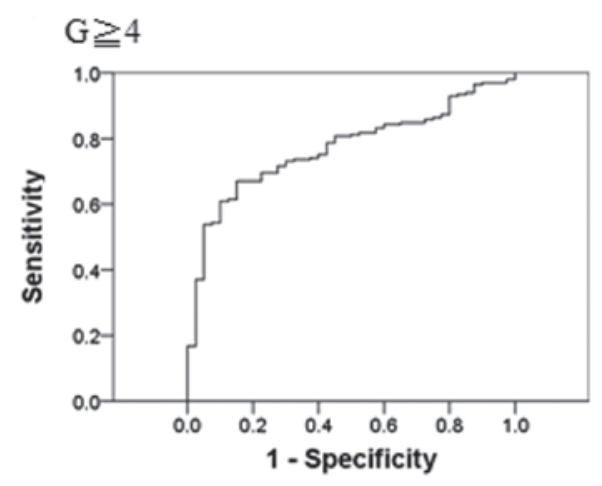

C

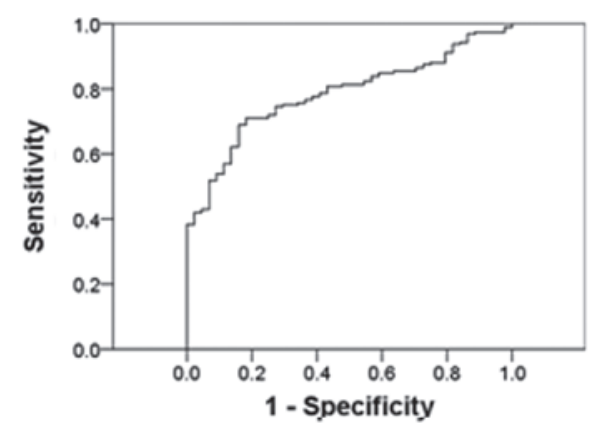

B

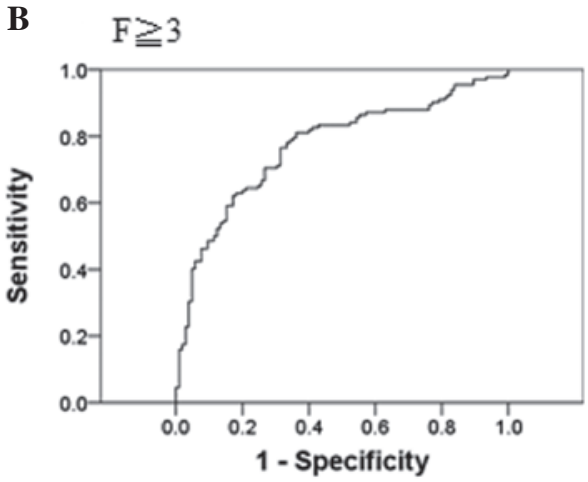

D

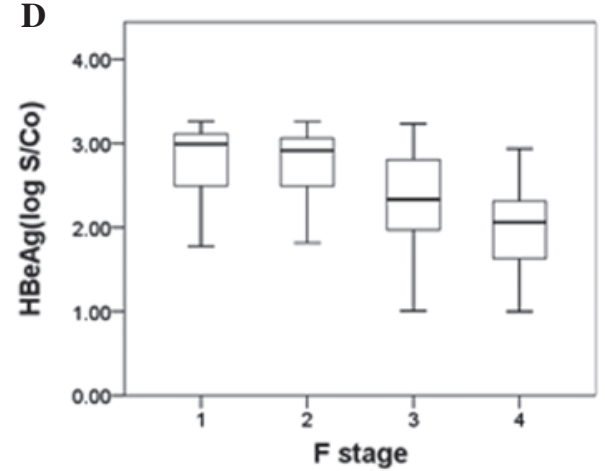

Figure 2. (A-C) Receiver operating characteristic curves of performance of HBeAg levels in distinguishing the different stages of inflammation and fibrosis. (D) Median HBeAg levels ( $\log 10 \mathrm{IU} / \mathrm{ml}$ ) at liver inflammation stages F1 to F4. G, liver inflammation stages; F, liver fibrosis stages; HBsAg, hepatitis B surface antigen; HBeAg, hepatitis B ' $\mathrm{e}$ ' antigen. 
Table IV. Validity of noninvasive models for prediction of cirrhosis and comparison with IC-model.

\begin{tabular}{lccccccc}
\hline $\begin{array}{l}\text { Fibrosis } \\
\text { test }\end{array}$ & $\begin{array}{c}\text { Cut-off } \\
\text { point }\end{array}$ & $\begin{array}{c}\text { Sensitivity } \\
(\%)\end{array}$ & $\begin{array}{c}\text { Specificity } \\
(\%)\end{array}$ & $\begin{array}{c}\text { PPV } \\
(\%)\end{array}$ & $\begin{array}{c}\text { NPV } \\
(\%)\end{array}$ & $\begin{array}{c}\text { AUC } \\
(95 \% \text { CI })\end{array}$ & $\begin{array}{c}\text { P-value } \\
\text { (vs. IC-model) }\end{array}$ \\
\hline IC-model & -1.89 & 90.91 & 74.48 & 44.94 & 97.29 & $0.87(0.81-0.93)^{\mathrm{a}}$ & \\
FI & $-1.5 \times 10^{9}$ & 59.09 & 84.97 & 32.13 & 94.51 & $0.74(0.65-0.83)^{\mathrm{a}}$ & 0.009 \\
AP index & $1.7 \times 10^{-10}$ & 84.09 & 64.06 & 47.85 & 91.12 & $0.78(0.71-0.86)^{\mathrm{a}}$ & 0.041 \\
\hline
\end{tabular}

PPV, positive predictive value; NPV, negative predictive value; FI, fibrosis index; AP index, age-platelet index; IC, immune clearance; AUC, area under the curve.

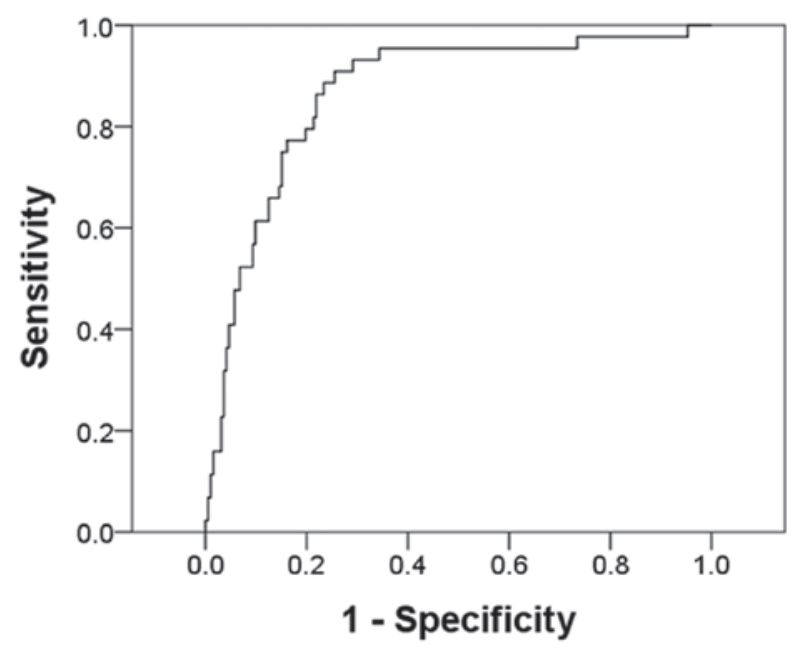

Figure 3. Receiver operating characteristic curve for the immune clearance model.

variables identified by multivariate logistic regression were used to construct the following formula for the predictor model: Predictor model $=-0.614+0.065 \times \log ($ age $)-$ $0.623 x \log (\mathrm{HBsAg})-0.631 \mathrm{x} \log (\mathrm{HBeAg})+9.302 \mathrm{x}$ $\log$ (INR). Fig. 3 shows the ROC curve from which the cut-off point was selected. The AUC was 0.87 ( $\mathrm{P}<0.001,95 \%$ CI: 0.81 to 0.93$)$. The cut-off point was designated as the probability of $(-1.89)$. Table IV presents the sensitivity and specificity of the model. Using this cut-off point, the likelihood of cirrhosis with a $88.64 \%$ sensitivity and $78.24 \%$ specificity could be predicted. The PPV for predicting patients with cirrhosis was 48.15\%; however, the NPV for patients not having cirrhosis was $96.79 \%$. Fig. 4 shows the ROC curves of the IC model, APRI, age-AST model, FIB-4, FI, and AP index for predicting cirrhosis. The results indicated that the APRI (AUC $=0.57$, $\mathrm{P}=0.162$ ), age-AST model ( $\mathrm{AUC}=0.57, \mathrm{P}=0.177$ ) and FIB-4 (AUC $=0.58, \mathrm{P}=0.115$ ) were not good predictor models for patients with cirrhosis. Further comparisons of the IC model with the FI and AP index are shown in Table IV. The IC model had a significantly improved predictive performance, as demonstrated by the largest AUC $(\mathrm{P}<0.05)$.

\section{Discussion}

In the present study, both the HBsAg and HBeAg were evaluated as biomarkers to predict fibrosis in patients with CHB. There was a negative and indirect correlation between the HBsAg

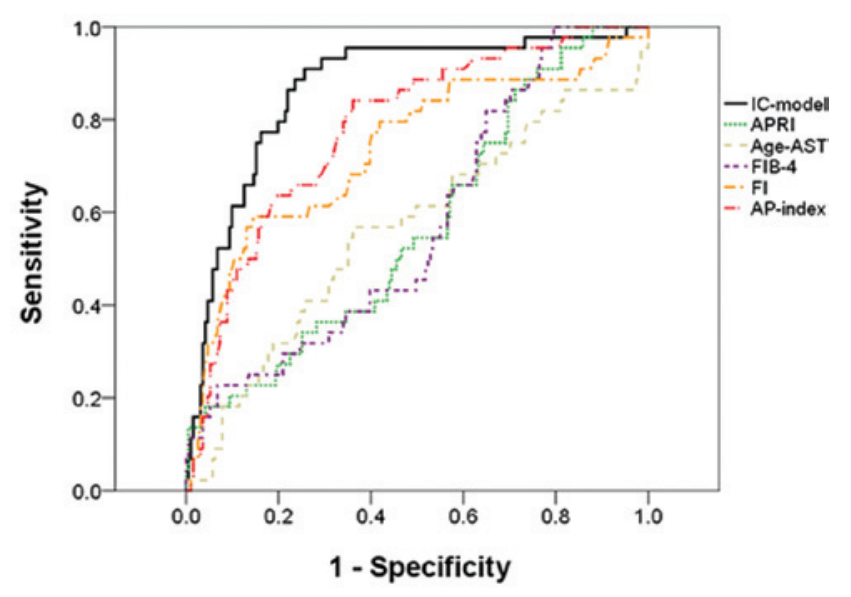

Figure 4. Receiver operating characteristic curve of the IC model (black line), APRI (dotted green line), age-AST model (dashed olive line), FIB-4 (dashed purple line), FI (dot-dashed orange line) and AP index (dot-dashed red line) for predicting cirrhosis. IC, immune clearance; FI, fibrosis index; AP index, age-platelet index; AST, aspartate aminotransferase; APRI, AST to platelet ratio index.

and $\mathrm{HBe} A g$ levels and liver inflammation and fibrosis in CHB patients who were in the IC phase. A liver pathology-predicting model (the IC model), which accurately and reliably predicted cirrhosis, was developed for this study population.

The current understanding of the natural course of CHB infection has been expanded by numerous research observations, including: i) HBV replication persists throughout the course of chronic HBV infection, ii) the host immune response has a pivotal role in HBV-associated liver injury, and iii) the balance between the host immune response and HBV replication is dynamic. The IT, IC, LR and ENH phases of CHB are classified based on biochemical, serological and virological characteristics, including serum ALT levels, HBeAg serostatus and HBV DNA levels $(30,31)$. Patients in the IC phase are $\mathrm{HBsAg}$ positive for at least six months, and $\mathrm{HBeAg}$-positive patients have serum levels of HBV DNA $\geq 2000 \mathrm{IU} / \mathrm{ml}$ $\left(\sim 10^{4}\right.$ copies $\left./ \mathrm{ml}\right)$ and ALT levels at least twice the ULN $(6,7)$. Patients with late childhood, adolescence or adult-acquired CHB infection usually present in the immunoactive phase with $\mathrm{HBeAg}$ positive chronic hepatitis and exhibit elevated serum ALT and moderate to severe necroinflammation with variable amounts of fibrosis in the liver biopsy.

Previous studies have reported that the median levels of HBsAg among Asian and European carriers in the IC phase 
were 4.03 and $4.37 \log \mathrm{IU} / \mathrm{ml}$, respectively $(7,9)$. These data were consistent with the present study, which showed a median of HBsAg levels of $3.94 \log \mathrm{IU} / \mathrm{ml}$ among the CHB patients of the present study in the IC phase.

Levels of albumin, cholinesterase, cholesterol, HBsAg and HBeAg all showed a negative correlation with liver inflammation and fibrosis. These data were consistent with a recent report showing that low serum HBsAg levels were associated with moderate to severe fibrosis in $\mathrm{HBeAg}$-positive $\mathrm{CHB}$ patients and a serum HBsAg cutoff of $3.85 \mathrm{log} \mathrm{IU} / \mathrm{ml}$ was predictive of fibrosis severity in patients with hepatitis $\mathrm{B}$ or C virus (20). Previous reports have shown a strong correlation between HBsAg and HBV DNA among Asian patients during the immune clearance phase (9). Based on these studies, it will be valuable to compare the efficacy of $\mathrm{HBsAg}$ and $\mathrm{HBeAg}$ levels as non-invasive markers of liver inflammation and fibrosis stages to that of HBV DNA.

The IC phase is characterized by flares of aminotransferases, which occur as a result of immune-mediated lysis of infected hepatocytes secondary to increased T-cell responses to $\mathrm{HBcAg}$ and $\mathrm{HBeAg}(32,33)$. The duration of the IC phase, and the frequency and severity of flares, have been shown to correlate with the risk of cirrhosis (34). The IC phase represents the period of symptomatic hepatitis, which lasts for 3-4 weeks in patients with acute HBV infection. In patients with chronic disease, the IC phase may persist for 10 or more years, during which severe necroinflammation may result in morphologically apparent fibrosis, leading to cirrhosis $(35,36)$. In the present study, a significant decrease in the HBsAg and HBeAg levels with increasing liver damage suggested that liver injury is mediated by the host cellular response to small epitopes of HBV proteins, which is consistent with previous reports (36). HBsAg and HBeAg levels reflected the role of the immune system in CHB patients in the present study; however, the possibility of representation bias in this single-centered study should be acknowledged, since the biopsied patients were not randomly selected.

Although liver biopsy is currently the gold standard in assessing liver histology, it is an invasive and expensive procedure, making it imperative to develop accurate and non-invasive alternatives. ROC curve analysis was used in the present study to show that the AUC for $\mathrm{HBsAg}$ was $0.74(\mathrm{~F} \geq 4)$ and the AUC for $\mathrm{HBeAg}$ was $0.78(\mathrm{~F} \geq 4)$. HBsAg and $\mathrm{HBeAg}$ levels were predicted to be indirectly associated with the stage of fibrosis in CHB patients in the IC phase by: i) A previous report showing that age and INR were determinants of fibrosis progression in $\mathrm{CHB}$ and $\mathrm{CHC}$ patients (37) and ii) a model consisting of a combination of four variables ( $\mathrm{HBsAg}, \mathrm{HBeAg}$, age and INR) to distinguish between patients with and without cirrhosis. A number of studies have explored the feasibility of using non-invasive tests to predict cirrhosis. These include the Fibroscan and FibroTest-ActiTest, galactose and methacetin breath tests, TE, fibrotest, cirrhosis discriminant score, AST/ALT ratio, APRI, FIB-4 and AP index (24-29). However, certain tests are impractical to use in a clinical setting since they require expensive instrumentation or may use less common biochemical markers, including $\alpha 2$-macroglobulin, haptoglobin and apolipoprotein A1, or the use of specialized software for computations. By contrast, the IC model generated in the present study combined routinely available laboratory test results along with serum $\mathrm{HBsAg}$ and $\mathrm{HBeAg}$ levels to accurately predict cirrhosis in treatment-naïve patients who are in the IC phase of HBV infection. The IC model predicted cirrhosis in CHB patients in the IC phase, with an AUC of 0.87 , a sensitivity of $88.64 \%$, a specificity of $78.24 \%$, a PPV of $48.15 \%$ and an NPV of $96.79 \%$. This data suggested that a combination of routinely used markers, together with HBsAg and $\mathrm{HBeAg}$ levels, is able to reliably and accurately predict the probability of liver cirrhosis. The IC model also exhibited a significantly higher predictive performance as compared with the FI and the AP index models.

It has been documented for numerous years that the transition from the IT phase of an HBV infection to the IC phase is caused by the activation of the immune system against virus replication, which causes necro-inflammation within the liver. If this immune reaction is short and effective, it may lead to: i) Immune clearance of serum $\mathrm{HBeAg}$ and anti-HBeAg seroconversion with complete recovery and eventual HBsAg loss and anti-HBsAg seroconversion; ii) the transition to the inactive $\mathrm{HBsAg}$ positive, $\mathrm{HBeAg}$ negative and anti-HBeAg positive carrier status associated with the clearance of HBV-associated liver disease, namely recovery from $\mathrm{HBeAg}$ positive CHB. If the immune reaction is long lasting and ineffective, it may lead to anti-HBeAg seroconversion and persistence of liver inflammation. This can result in progressive fibrosis, eventually evolving to cirrhosis and HCC through the progression of $\mathrm{HBeAg}$ positive CHB or reactivation of the HBV infection, namely $\mathrm{HBeAg}$ negative $\mathrm{CHB}$. The decline of serum HBsAg and $\mathrm{HBeAg}$ can therefore be associated with either complete recovery and cure or progressive liver disease.

Cross-sectional retrospective studies require longitudinal follow-up, since an HBV-infection is a highly dynamic disease. One major limitation of the present study is that all consecutive patients in the IC phase were not followed up for long enough (five years). With a longer-term follow-up, all consecutive patients would have been divided into: i) Those who seroconverted to anti-HBeAg, eventually progressing to the inactive $\mathrm{HBsAg}$ carrier condition or to $\mathrm{HBeAg}$ negative $\mathrm{CHB}$, and ii) those who did not persist in a progressive $\mathrm{HBeAg}$ positive CHB. The analysis of the baseline levels of HBsAg and $\mathrm{HBeAg}$ and their ratios would have been correlated with outcomes, histology and baseline, and possibly histology or a surrogate test of liver fibrosis (fisbroscan, fibrotest APRI, other) at the end of several years of follow-up. Furthermore, the current data did not validate the non-invasive model in a separate group of patients. The clinical relevance of these findings is difficult to observe, since all patients are required to later be administered antiviral therapy according to European Association for the Study of the Liver (EASL) guidelines (38).

In conclusion, $\mathrm{HBsAg}$ and $\mathrm{HBeAg}$ levels exhibited a negative correlation with liver inflammation and fibrosis in patients with CHB in the IC phase, and can be considered as potential markers which predict significant necroinflammation and fibrosis. HBsAg and HBeAg levels may additionally be used as a non-invasive predictors of the degree of hepatic fibrosis. The present findings have important implications, particularly in patients who refuse to undergo liver biopsy or who have contraindications. The IC model, which uses routinely assessed markers in combination with $\mathrm{HBsAg}$ and $\mathrm{HBeAg}$ 
levels, reliably predicted the probability of liver cirrhosis. It is important to validate these data in larger, prospective studies.

\section{References}

1. Liang X, Bi S, Yang W, et al: Epidemiological serosurvey of hepatitis B in China - declining HBV prevalence due to hepatitis B vaccination. Vaccine 27: 6550-6557, 2009.

2. Lok AS: Chronic hepatitis B. N Engl J Med 346: 1682-1683, 2002.

3. Shi $\mathrm{YH}$ and Shi $\mathrm{CH}$ : Molecular characteristics and stages of chronic hepatitis B virus infection. World J Gastroenterol 15: 3099-3105, 2009.

4. Blumberg BS, Alter HJ and Visnich S: A "new" antigen in leukemia sera. JAMA 191: 541-546, 1965.

5. Rotman Y, Brown TA and Hoofnagle JH: Evaluation of the patient with hepatitis B. Hepatology 49: S22-27, 2009.

6. Cornberg M, Protzer U, Dollinger MM, et al: The German guideline for the management of hepatitis $B$ virus infection: short version. J Viral Hepat 15: 1-21, 2008.

7. Jaroszewicz J, Calle Serrano B, Wursthorn K, et al: Hepatitis B surface antigen (HBsAg) levels in the natural history of hepatitis B virus (HBV)-infection: a European perspective. J Hepatol 52: 514-522, 2010

8. WhiteheadTP,ThorpeGHG,CarterTJN,GroucuttCandKrickaLJ: Enhanced luminescence procedure for sensitive determination of peroxidase labelled conjugates in immunoassay. Nature 305: $158,1983$.

9. Nguyen T, Desmond P and Locarnini S: The role of quantitative hepatitis B serology in the natural history and management of chronic hepatitis B. Hepatol Int 3: 5-15, 2009.

10. Dwivedi M, Misra SP, Misra V, et al: Seroprevalence of hepatitis B infection during pregnancy and risk of perinatal transmission. Indian J Gastroenterol 30: 66-71, 2011.

11. Ott JJ, Stevens GA and Wiersma ST: The risk of perinatal hepatitis B virus transmission: hepatitis $\mathrm{B}$ e antigen $(\mathrm{HBeAg})$ prevalence estimates for all world regions. BMC Infect Dis 12: 131, 2012.

12. Chen $\mathrm{CH}$, Lee $\mathrm{CM}$, Hung $\mathrm{CH}$, et al: Hepatitis $\mathrm{B}$ virus genotype $\mathrm{B}$ results in better immediate, late and sustained responses to peginterferon-alfa in hepatitis-B-e-antigen-positive patients J Gastroenterol Hepatol 26: 461-468, 2011.

13. Perrillo R, Mimms L, Schechtman K, Robbins D and Campbell C: Monitoring of antiviral therapy with quantitative evaluation of HBeAg: a comparison with HBV DNA testing. Hepatology 18: 1306-1312, 1993.

14. Yen YH, Lu SN, Chen CH, et al: Changes in serum hepatitis $\mathrm{B}$ e antigen $(\mathrm{HBeAg})$ levels associated with the emergence of YMDD mutants in HBeAg non-seroconverted patients during lamivudine therapy. Liver Int 27: 1349-1355, 2007.

15. Cadranel JF, Rufat P and Degos F: Practices of liver biopsy in France: results of a prospective nationwide survey. For the Group of Epidemiology of the French Association for the Study of the Liver (AFEF). Hepatology 32: 477-481, 2000.

16. Chan HL, Wong VW, Wong GL, et al: A longitudinal study on the natural history of serum hepatitis B surface antigen changes in chronic hepatitis B. Hepatology 52: 1232-1241, 2010.

17. Gupta E, Kumar A, Choudhary A, Kumar M and Sarin SK: Serum hepatitis B surface antigen levels correlate with high serum HBV DNA levels in patients with chronic hepatitis B: a cross-sectional study. Indian J Med Microbiol 30: 150-154, 2012.

18. Seto WK, Wong DK, Fung J, et al: High hepatitis B surface antigen levels predict insignificant fibrosis in hepatitis $\mathrm{B}$ e antigen positive chronic hepatitis B. PLoS One 7: e43087, 2012.
19. Liu SQ, Zhu XJ, Sun XH, Li M and Gao YQ: Characteristic of liver pathology in HBeAg-positive and HBeAg-negative chronic hepatitis B patients with mildly elevated ALT. Zhonghua Gan Zang Bing Za Zhi 20: 348-352, 2012 (In Chinese).

20. Martinot-Peignoux M, Carvalho-Filho R, Lapalus M, et al: Hepatitis B surface antigen serum level is associated with fibrosis severity in treatment-naïve, e antigen-positive patients. J Hepatol 58: 1089-1095, 2013.

21. Liaw, YF, Kao JH, Piratvisuth T et al: Asian-Pacific consensus statement on the management of chronic hepatitis B: a 2012 update. Hepatol Int 6: 531-561, 2012.

22. Scheuer PJ, Standish RA and Dhillon AP: Scoring of chronic hepatitis. Clin Liver Dis 6: 335-47, 2002.

23. The programme of prevention and cure for viral hepatitis. In: Parasitology ASoIDa, Medical aCSoHoC, Association, Eds. Zhonghua Ganzangbing Zazhi. Vol. 8, 324-329, 2000.

24. Kim SM, Sohn JH, Kim TY, et al: Comparison of various noninvasive serum markers of liver fibrosis in chronic viral liver disease. Korean J Hepatol 15: 454-463, 2009 (In Korean).

25. Lee IC, Chan CC, Huang YH, et al: Comparative analysis of noninvasive models to predict early liver fibrosis in hepatitis $\mathrm{B}$ e Antigen-negative Chronic Hepatitis B. J Clin Gastroenterol 45: 278-285, 2011.

26. Tong MJ, Hsu L, Hsien C, et al: A comparison of hepatitis B viral markers of patients in different clinical stages of chronic infection. Hepatol Int 4: 516-522, 2010.

27. Park SH, Kim CH, Kim DJ, et al: Development and validation of a model to predict advanced fibrosis in chronic hepatitis B virus-infected patients with high viral load and normal or minimally raised ALT. Dig Dis Sci 56: 1828-1834, 2011.

28. Poynard T, Ngo Y, Marcellin P, et al: Impact of adefovir dipivoxil on liver fibrosis and activity assessed with biochemical markers (FibroTest-ActiTest) in patients infected by hepatitis B virus. J Viral Hepat 16: 203-213, 2009.

29. Stibbe KJ, Verveer C, Francke J, et al: Comparison of non-invasive assessment to diagnose liver fibrosis in chronic hepatitis B and C patients. Scand J Gastroenterol 46: 962-972, 2011.

30. Liaw YF, Leung N, Kao JH, et al: Asian-Pacific consensus statement on the management of chronic hepatitis B: a 2008 update. Hepatol Int 2: 263-283, 2008.

31. European Association For The Study Of The Liver: EASL Clinical Practice Guidelines: management of chronic hepatitis B. J Hepatol 50: 227-242, 2009.

32. Tsai SL, Chen PJ, Lai MY, et al: Acute exacerbations of chronic type B hepatitis are accompanied by increased $\mathrm{T}$ cell responses to hepatitis B core and e antigens. Implications for hepatitis B e antigen seroconversion. J Clin Invest: 89: 87-96, 1992.

33. Chu CM and Liaw YF: Intrahepatic distribution of hepatitis B surface and core antigens in chronic hepatitis B virus infection. Hepatocyte with cytoplasmic/membranous hepatitis B core antigen as a possible target for immune hepatocytolysis. Gastroenterology 92: 220-225, 1987.

34. McMahon BJ, Holck P, Bulkow L and Snowball M: Serologic and clinical outcomes of 1536 Alaska Natives chronically infected with hepatitis B virus. Ann Intern Med 135: 759-768, 2001.

35. Friedman SL: Liver fibrosis - from bench to bedside. J Hepatol 38: S38-S53, 2003

36. Lee WM: Hepatitis B virus infection. N Engl J Med 337: 1733-1745, 1997.

37. Imperial JC: Natural history of chronic hepatitis B and C. J Gastroenterol Hepatol 14: S1-S5, 1999.

38. European Association for the Study of the Liver. EASL clinical practice guidelines: Management of chronic hepatitis B virus infection. J Hepatol 57: 167-185, 2012. 